\title{
National Trends in Smoking Cessation Medication Prescriptions for Smokers With Chronic Obstructive Pulmonary Disease in the United States, 2007-2012
}

\author{
Min Ji Kwak ${ }^{1,2}$, Jongoh Kim³ ${ }^{3}$ Viraj Bhise ${ }^{2,4}$, Tong Han Chung ${ }^{5}$, Gabriela Sanchez Petitto ${ }^{1}$ \\ ${ }^{1}$ Department of Internal Medicine, McGovern Medical School, University of Texas Health Science Center at Houston, Houston, TX, USA; ${ }^{2}$ Department \\ of Management Policy and Community Health, University of Texas School of Public Health, Houston, TX, USA; ${ }^{3}$ Department of Medicine, Baylor \\ College of Medicine, Houston, TX, USA; ${ }^{4}$ Department of Internal Medicine, John A Burns School of Medicine, University of Hawaii at Manoa, \\ Honolulu, HI, USA; ${ }^{5}$ Healthcare Transformation Initiatives, McGovern Medical School, University of Texas Health Science Center at Houston, \\ Houston, TX, USA
}

Objectives: Smoking cessation decreases morbidity and mortality due to chronic obstructive pulmonary disease (COPD). Pharmacotherapy for smoking cessation is highly effective. However, the optimal prescription rate of smoking cessation medications among smokers with COPD has not been systemically studied. The purpose of this study was to estimate the national prescription rates of smoking cessation medications among smokers with COPD and to examine any disparities therein.

Methods: We conducted a retrospective study using National Ambulatory Medical Care Survey data from 2007 to 2012. We estimated the national prescription rate for any smoking cessation medication (varenicline, bupropion, and nicotine replacement therapy) each year. Multiple survey logistic regression was performed to characterize the effects of demographic variables and comorbidities on prescriptions.

Results: The average prescription rate of any smoking cessation medication over 5 years was $3.64 \%$. The prescription rate declined each year, except for a slight increase in 2012: 9.91\% in 2007, 4.47\% in 2008, 2.42\% in 2009, 1.88\% in 2010, 1.46\% in 2011, and 3.67\% in 2012. Hispanic race and depression were associated with higher prescription rates (odds ratio [OR], 5.15; 95\% confidence interval [Cl], 1.59 to 16.67 and $\mathrm{OR}, 2.64 ; 95 \% \mathrm{Cl}, 1.26$ to 5.51 , respectively). There were no significant differences according to insurance, location of the physician, or other comorbidities. The high OR among Hispanic population and those with depression was driven by the high prescription rate of bupropion.

Conclusions: The prescription rate of smoking cessation medications among smokers with COPD remained low throughout the study period. Further studies are necessary to identify barriers and to develop strategies to overcome them.

Key words: Chronic obstructive pulmonary disease, Smoking cessation, Varenicline, Bupropion, Tobacco use cessation products, Nicotine

Received: May 25, 2018 Accepted: August 23, 2018

Corresponding author: Min Ji Kwak, MD, MS

Department of Internal Medicine, McGovern Medical School, University of Texas Health Science Center at Houston, 6431 Fannin Street, Houston, TX 77030, USA

E-mail: dr.min.ji.kwak@gmail.com

This is an Open Access article distributed under the terms of the Creative Commons Attribution Non-Commercial License (http://creativecommons.org/licenses/bync/4.0// which permits unrestricted non-commercial use, distribution, and reproduction in any medium, provided the original work is properly cited.

\section{INTRODUCTION}

Chronic obstructive pulmonary disease (COPD) is one of the leading causes of morbidity and mortality worldwide, and has been listed as the third most common cause of death by the World Health Organization [1]. Both the prevalence and mortality of COPD are rapidly increasing [1-4]. In 2007, 5.8\% of adults aged 48 to 64 years had COPD [4]. Due to its chronic 
and progressive disease course, the economic burden of COPD to patients and to society as a whole is substantial [3-6]. In 2008 , the estimated total cost of COPD was $\$ 68$ billion in the US, of which direct costs accounted for $\$ 53.7$ billion [2]. The annual COPD-related cost for an individual with commercial insurance was estimated as $\$ 3949$ in 2006 [4].

A large number of studies have been conducted to identify interventions to decrease the morbidity and mortality of COPD. Smoking cessation is one of the most effective interventions to decrease morbidity in COPD patients, as measured in terms of improvements in pulmonary function tests, fewer respiratory symptoms, and a lower hospitalization rate, as well as reduced mortality [7]. However, studies have shown that $30-40 \%$ of symptomatic COPD patients still smoke [8]. Various approaches to achieve smoking cessation have been proposed, including counseling, behavioral interventions, pharmacotherapy, and combinations thereof $[9,10]$. Pharmacotherapy, including varenicline, bupropion, and nicotine replacement treatment (NRT), is more effective than behavioral intervention alone [10-12]. A review article by Tønnesen [13] reported a successful rate of smoking cessation with pharmacotherapy among smokers with COPD. Considering that smoking cessation has significant benefits on lowering morbidity and mortality, and pharmacotherapy is known to be more effective for smoking cessation than behavioral therapy alone, the prescription of such medications for smokers with COPD should be highly recommended in the outpatient setting. However, few studies have reported whether these medications are prescribed adequately to current smokers with COPD. Previous studies have shown various prescription rates (8.8$16.9 \%$ ) in different healthcare settings [14-17]. Kim and Jeong [18] reported a smoking cessation medication prescription rate of $20 \%$ at a special smoking cessation clinic, but this proportion was for the general population, not COPD patients.

The purpose of this study was to estimate the national prescription rate of smoking cessation medications in smokers with COPD and to identify any differences in prescription rate according to patient characteristics.

\section{METHODS}

\section{Data}

We conducted a retrospective observational study using the National Ambulatory Medical Care Survey (NAMCS) database from 2007 to 2012. The NAMCS is a national survey conducted by the Centers for Disease Control and Prevention (CDC). Its goal is to provide objective and reliable information about ambulatory medical care service utilization in the US through a survey of visits to non-federally employed office-based physicians [19]. Randomly assigned physicians were surveyed about random samples of visits and the patients' demographic and clinical data were collected to construct an annual dataset for national estimation purposes. Institutional review board approval was not needed since the NAMCS data are publicly accessible. NAMCS data do not contain any identifiable patient information.

\section{Selection of Eligible Subjects}

The NAMCS provides 3 diagnostic codes per each patient. If a patient had any diagnostic code for COPD, then the patient was selected. We used the International Classification of Diseases, Ninth Revision, Clinical Modification codes 491, 492, and 496. Additionally, if a patient was known to have COPD by the physician, then the patient was also selected. Active smokers were identified using the survey question, "Does your patient smoke cigarettes?" NAMCS also provides information on up to 10 medications that the patient was taking. If any of the 3 medications of interest - varenicline, bupropion, and NRTwas listed, it was considered that the patient had been prescribed a smoking cessation medication. We used population weights that were provided by the CDC to generate a national estimate [19].

\section{Variables}

NAMCS provides data on demographic variables such as age, sex, ethnicity and insurance information, as well as comorbidities including depression, asthma, cancer, cardiovascular disease, chronic renal failure, congestive heart failure, diabetes, hyperlipidemia, hypertension, ischemic heart disease, obesity, osteoporosis and COPD. NAMCS also contains information about whether the physician's location of practice was a metropolitan area or not.

\section{Statistical Analysis}

We first estimated the total number of COPD patients and active smokers in visits each year using survey tabulate analysis. The Pearson chi-square test was conducted to explore changes over time. We did not use the Cochran-Armitage test for trend, as we employed a survey tabulate analysis. We then conducted multiple survey logistic regression to estimate 
odds ratios (ORs) for the prescription of smoking cessation medications using demographic variables and comorbidities. Stata/IC version 14.2 (StataCorp., College Station, TX, USA) was used for all statistical analyses. The $p$-values $<0.05$ were considered to indicate statistical significance.

\section{RESULTS}

The total estimated number of adult visits over the 6 years of the study was 4744275777 . Among them, 215935667 (4.55\% of all adult visits) were made by patients estimated to have COPD, and 53107842 of the COPD patients (24.59\%) were estimated to be active smokers. Among the active smokers with COPD, the average prescription rate for any smoking cessation medication during the study years was $3.64 \%$. The percentage of COPD patients and the percentage of active smokers have not changed over time (Table 1). However, the prescription rate declined each year, except for a slight increase in 2012: $9.91 \%$ in $2007,4.47 \%$ in $2008,2.42 \%$ in $2009,1.88 \%$ in $2010,1.46 \%$ in 2011, and $3.67 \%$ in 2012 (Figure 1). Among the 3 medications, varenicline was the most commonly prescribed, followed by bupropion and NRT (Table 1). Multiple survey logistic regression showed that the year of the survey was associated with a lower prescription rate $(\mathrm{OR}, 0.60$ for each year; $95 \% \mathrm{Cl}, 0.44$ to 0.81 ), which means that the likelihood of prescription decreased over time. Hispanic race and depression were associated with higher prescription rates (OR, 5.15; 95\% $\mathrm{Cl}, 1.59$ to 16.67 and $\mathrm{OR}, 2.64 ; 95 \% \mathrm{Cl}, 1.26$ to 5.51 , respectively). There were no significant differences in the prescription of smoking cessation medications according to insurance, loca- tion of the physician, or other comorbidities (Table 2).

When analyzed for each smoking cessation medication, Asian or other racial groups and patients with depression were less likely to be prescribed varenicline $(\mathrm{OR}, 0.12 ; 95 \% \mathrm{Cl}, 0.01$ to 0.94 and $\mathrm{OR}, 0.26 ; 95 \% \mathrm{Cl}, 0.07$ to 0.95 , respectively). $\mathrm{Pa}$ tients with "other" insurance (including worker's compensation, unknown, unclassified, or blank) and patients with hyperlipidemia were more likely to be prescribed varenicline $(\mathrm{OR}$, 4.16; $95 \% \mathrm{Cl}, 1.03$ to 16.85 and $\mathrm{OR}, 2.91 ; 95 \% \mathrm{Cl}, 1.18$ to 7.16 , respectively). Bupropion was more likely to be prescribed to those with depression, chronic renal failure, and obesity (OR, $16.47 ; 95 \% \mathrm{Cl}, 6.03$ to $44.99 ; \mathrm{OR}, 11.70 ; 95 \% \mathrm{Cl}, 2.08$ to 65.89 ; and $\mathrm{OR}, 3.70 ; 95 \% \mathrm{Cl}, 1.06$ to 12.96 , respectively). Moreover, Hispanic patients were more likely to be prescribed bupropion (OR, 8.29; 95\% Cl, 2.50 to 27.48). However, bupropion was the only medication that showed a significant negative associa-

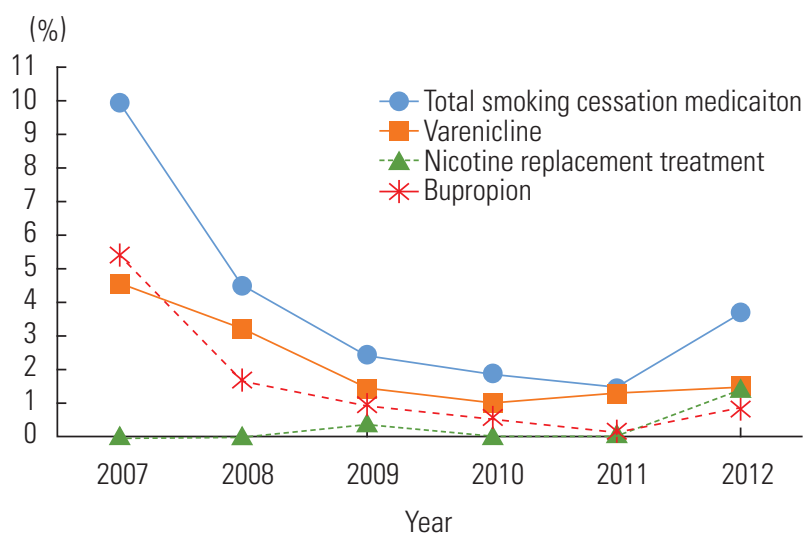

Figure 1. Smoking cessation medications prescription rate among smokers with chronic obstructive pulmonary disease.

Table 1. Smoking cessation medications prescription rate among smokers with COPD

\begin{tabular}{|c|c|c|c|c|c|c|c|c|c|c|}
\hline \multirow{2}{*}{ Year } & \multirow{2}{*}{\multicolumn{2}{|c|}{$\begin{array}{l}\text { Currently smoking among } \\
\text { COPD patients }\end{array}$}} & \multicolumn{8}{|c|}{ Prescribed } \\
\hline & & & \multicolumn{2}{|c|}{$\begin{array}{c}\text { Any smoking cessation } \\
\text { medication }\end{array}$} & \multicolumn{2}{|c|}{ Varenicline } & \multicolumn{2}{|c|}{ Bupropion } & \multicolumn{2}{|c|}{ NRT } \\
\hline 2007 & 239 & 7317404 (23.43) & 21 & 725342 (9.91) & 10 & 330529 (4.52) & 11 & $394813(5.40)$ & 0 & $0(0.00)$ \\
\hline 2009 & 268 & 9071960 (21.95) & 12 & 219571 (2.42) & 5 & $128074(1.41)$ & 4 & 80134 (0.88) & 4 & $31141(0.34)$ \\
\hline 2010 & 279 & 10319242 (27.42) & 10 & 194465 (1.88) & 3 & 102039 (0.99) & 2 & $58141(0.56)$ & 5 & 34285 (0.33) \\
\hline 2011 & 302 & 10539997 (26.45) & 7 & $154100(1.46)$ & 5 & 135932 (1.29) & 1 & $16256(0.15)$ & 1 & $1912(0.02)$ \\
\hline 2012 & 799 & 8312611 (25.94) & 30 & 304678 (3.67) & 15 & 122286 (1.47) & 7 & $69963(0.84)$ & 8 & 112429 (1.35) \\
\hline Total & 2140 & 53107842 (24.59) & 98 & 1935253 (3.64) & 50 & 1059345 (1.99) & 32 & $742434(1.40)$ & 18 & $179767(0.34)$ \\
\hline
\end{tabular}

COPD, chronic obstructive pulmonary disease; NRT, nicotine replacement therapy.

${ }^{*} p<0.05,{ }^{* *} p<0.01$. 
Table 2. Odds ratio of smoking cessation medication prescription among smokers with chronic obstructive pulmonary disease

\begin{tabular}{|c|c|c|c|c|c|}
\hline \multicolumn{2}{|l|}{ Variables } & Any & Varenicline & Bupropion & NRT \\
\hline \multicolumn{2}{|l|}{ Year } & $0.60(0.44,0.81)^{*}$ & $0.71(0.49,1.04)$ & $0.39(0.26,0.61)^{*}$ & $1.15(0.70,1.90)$ \\
\hline \multicolumn{2}{|l|}{ Age } & $0.99(0.96,1.01)$ & $0.99(0.96,1.02)$ & $0.97(0.93,1.02)$ & $0.96(0.93,0.99)^{*}$ \\
\hline \multicolumn{2}{|l|}{ Male } & $1.69(0.79,3.62)$ & $1.21(0.48,3.05)$ & $1.40(0.44,4.46)$ & $1.36(0.63,2.96)$ \\
\hline \multirow[t]{4}{*}{ Race } & NH White & 1.00 (reference) & 1.00 (reference) & 1.00 (reference) & 1.00 (reference) \\
\hline & NH Black & $1.20(0.31,4.63)$ & $0.95(0.14,6.30)$ & $1.79(0.16,20.01)$ & $1.02(0.05,21.96)$ \\
\hline & Hispanic & $5.15(1.59,16.67)^{*}$ & $3.46(0.63,18.95)$ & $8.29(2.50,27.48)^{*}$ & N/A \\
\hline & Asian/others & $1.04(0.15,7.08)$ & $0.12(0.01,0.94)^{*}$ & $17.49(0.99,308.00)$ & $39.46(2.49,625.95)^{*}$ \\
\hline \multirow[t]{6}{*}{ Insurance } & Private & 1.00 (reference) & 1.00 (reference) & 1.00 (reference) & 1.00 (reference) \\
\hline & Medicare & $1.08(0.45,2.60)$ & $1.25(0.41,3.83)$ & $0.65(0.20,2.18)$ & $3.78(0.26,53.94)$ \\
\hline & Medicaid & $1.01(0.38,2.68)$ & $1.17(0.31,4.46)$ & $0.38(0.09,1.54)$ & $5.89(0.09,379.94)$ \\
\hline & Self-pay & $0.99(0.22,4.36)$ & $2.64(0.57,12.27)$ & $\mathrm{N} / \mathrm{A}$ & $\mathrm{N} / \mathrm{A}$ \\
\hline & No charge/charity & $3.24(0.16,66.10)$ & $\mathrm{N} / \mathrm{A}$ & $3.09(0.00,3485.79)$ & $\mathrm{N} / \mathrm{A}$ \\
\hline & Other & $1.43(0.33,6.17)$ & $4.16(1.03,16.85)^{*}$ & $0.04(0.00,0.32)^{*}$ & $1.23(0.03,53.06)$ \\
\hline \multicolumn{2}{|c|}{ Non-MSA of physician's location } & $0.85(0.37,1.96)$ & $0.27(0.06,1.18)$ & $1.89(0.55,6.54)$ & $3.48(0.11,107.60)$ \\
\hline \multirow[t]{13}{*}{ Comorbidity } & Depression & $2.64(1.26,5.51)^{*}$ & $0.26(0.07,0.95)^{*}$ & $16.47(6.03,44.99)^{*}$ & $0.49(0.05,4.64)$ \\
\hline & Other mental disease & $1.50(0.15,14.71)$ & $\mathrm{N} / \mathrm{A}$ & $5.20(0.48,56.01)$ & $3.78(0.27,52.74)$ \\
\hline & Asthma & $0.43(0.15,14.71)$ & $0.58(0.15,2.33)$ & $0.19(0.02,2.28)$ & $0.54(0.02,19.09)$ \\
\hline & Cancer & $0.74(0.26,2.09)$ & $0.48(0.07,3.20)$ & $1.44(0.26,8.10)$ & $1.40(0.07,26.35)$ \\
\hline & CVD & $0.28(0.04,2.13)$ & $0.55(0.06,4.68)$ & $\mathrm{N} / \mathrm{A}$ & $\mathrm{N} / \mathrm{A}$ \\
\hline & CRF & $1.21(0.22,6.49)$ & $\mathrm{N} / \mathrm{A}$ & $11.70(2.08,65.89)^{*}$ & $\mathrm{~N} / \mathrm{A}$ \\
\hline & $\mathrm{CHF}$ & $0.65(0.17,2.41)$ & $\mathrm{N} / \mathrm{A}$ & $0.66(0.11,3.87)$ & $\mathrm{N} / \mathrm{A}$ \\
\hline & Diabetes & $0.63(0.26,1.51)$ & $0.72(0.16,3.18)$ & $0.78(0.23,2.66)$ & N/A \\
\hline & Hyperlidipemia & $1.33(0.64,2.74)$ & $2.91(1.18,7.16)^{*}$ & $0.39(0.12,1.31)$ & $3.58(0.78,16.38)$ \\
\hline & Hypertension & $0.59(0.30,1.17)$ & $0.59(0.27,1.30)$ & $0.60(0.17,2.04)$ & $0.04(0.00,0.42)$ \\
\hline & IHD & $0.53(0.12,2.35)$ & $0.79(0.15,4.10)$ & $0.20(0.02,1.94)$ & N/A \\
\hline & Obesity & $2.03(0.69,6.01)$ & $0.80(0.13,5.10)$ & $3.70(1.06,12.96)^{*}$ & $0.52(0.04,6.89)$ \\
\hline & Osteoporosis & $0.19(0.02,1.42)$ & $0.03(0.00,0.29)$ & $0.54(0.04,8.19)$ & $\mathrm{N} / \mathrm{A}$ \\
\hline
\end{tabular}

Values are presented as odds ratio (95\% confidence interval).

NRT, nicotine replacement therapy; NH, non-Hispanic; MSA, metropolitan statistical area; CVD, cerebrovascular disease; CRF, chronic renal failure; CHF, congestive heart failure; IHD, ischemic heart disease; N/A. not applicable.

${ }^{*} p<0.05$.

tion with the year of prescription among the 3 medications $(\mathrm{OR}, 0.39 ; 95 \% \mathrm{Cl}, 0.26$ to 0.61$)$. It was less likely to be prescribed among those who had "other" insurance (OR, 0.04; $95 \% \mathrm{Cl}, 0.00$ to 0.32 ). Nicotine was the least prescribed medication, and its prescription rate did not show a significant relationship with the year of prescription. However, it was more likely to be prescribed among younger patients and the Asian/ other race population $(\mathrm{OR}, 0.96 ; 95 \% \mathrm{Cl}, 0.93$ to 0.99 and $\mathrm{OR}$, $39.46 ; 95 \% \mathrm{Cl}, 2.49$ to 625.95$)$.

\section{DISCUSSION}

Our study showed a very low and overall declining prescription rate of smoking cessation medications. The prescription rate was lower than previously described [14-16]. Vaidya et al. [15] reported an overall prescription rate of $8.8 \%$ for smoking cessation medications among smokers with COPD using a nationally representative dataset, the Medical Expenditure Panel Survey, from 2006 to 2010. Jarlenski et al. [16] reported a 16.0\% utilization of smoking cessation medications among Medicare beneficiaries who were smokers with COPD. Another study using Veterans Administration data showed a dispense rate of $16.9 \%$ for smoking cessation medications upon discharge from the hospital among patients who were actively smoking when admitted for acute COPD exacerbation $[14,17]$. The lower prescription rate in our study might be explained by the limitations of the NAMCS. Medication utilization, such as over-the-counter NRT, could have been under-reported on physician surveys. 
The prescription rate was calculated based on the number of visits, not the number of patients, which might have skewed the results. Regardless, it is discouraging that most studies have consistently shown low prescription rates for smoking cessation medications among smokers with COPD, despite its significant effectiveness. The optimal prescription rate for smokers with COPD has not yet been established. However, the results of this study should alert clinicians who are caring for patients with COPD to assist them in optimizing their care. Further study to establish the ideal prescription rate in smokers with COPD and to identify the barriers against prescribing smoking cessation medication should be conducted.

Table 1 shows that the overall frequency of the variables increased over time, but the actual percentage for current smokers among COPD patients decreased. This is thought to be due to the sampling methods. The NAMCS was built to allow researchers to estimate outcomes using weights considering the demographic or geographic information of the subjects. An increase in absolute frequency in sampled subjects does not always reflect an actual increase in population frequency. Furthermore, the NAMCS increased the sample size from 3819 physicians in 2011 to 15740 physicians in 2012 [19]. Hence, the frequency of the sampled subjects has increased, but the actual percentage of the smokers in COPD did not increase.

Interestingly, our study showed a higher OR for prescription of smoking cessation medications among the Hispanic population. However, Vaidya et al. [15] reported an OR of 0.70 for prescription of smoking cessation medications among the Hispanic population. Our analysis (Table 2) showed that this high OR was mainly driven by a high prescription rate of bupropion among the Hispanic population. The results presented in Table 2 also show a high NRT prescription rate among the Asian/others population. The authors believe that this finding was due to the very low prescription rate and small number of observations in the Hispanic and Asian/others populations. As Table 1 shows, the absolute number of prescriptions of bupropion and NRT was very low, so 1 or 2 positive values would have affected the overall OR. This is also reflected by very wide confidence intervals. Hence, it is possible that our analysis might have not captured an actual disparity. Again, future studies to investigate the potential of such a disparity are required. The results of this study also underscore that it may not even be possible to evaluate possible disparities in prescriptions due to the extremely low prescription rate.

In addition to the small values reported for the outcome variable, this study has another limitation; namely, that bupropion could have been prescribed for depression. Unfortunately, the NAMCS does not provide information explaining why patients were prescribed various medications. The authors tried to adjust for this confounding effect by using depression as one of the covariates in the regression analysis. Indeed, depression was closely related to the prescription of bupropion.

Nevertheless, it is noteworthy that the already very low prescription rate of smoking cessation medications declined significantly over the study period. One explanation could be the safety warning placed by US Food and Drug Administration (FDA) regarding varenicline and bupropion due to the risk of neuropsychiatric events for bupropion (2009) and varenicline (2007) [16], although there are no data to support the possibility of a relationship between the safety warning and the low prescription rate. Since then, the safety of varenicline and bupropion has been controversial. Importantly, in 2016, the FDA removed this warning based on a double-blinded randomized controlled trial $[12,20]$. At the time of the present study, post2012 data were not available. However, further studies should investigate trends in the usage of prescription smoking cessation medications after the FDA removed the warning. Considering the effectiveness of smoking cessation medications and the significant benefit of smoking cessation among COPD patients, the utilization of smoking cessation medications should be optimized. Further studies are warranted to continue analyzing trends in the prescription rate, to explore the barriers to prescription, and to design proper interventions to promote the prescription of appropriate smoking cessation medications in active smokers with COPD.

\section{ACKNOWLEDGEMENTS}

The authors would like to thank Dr. Eric J. Thomas for his comments and guidance for publication of this article.

\section{CONFLICT OF INTEREST}

The authors have no conflicts of interest associated with the material presented in this paper.

\section{ORCID}

Min Ji Kwak http://orcid.org/0000-0003-2778-3984

Jongoh Kim https://orcid.org/0000-0002-2660-3382 


\section{REFERENCES}

1. World Health Organization. The top 10 causes of death; 2018 May 24 [cited 2018 Sep 10]. Available from: http://www.who. int/en/news-room/fact-sheets/detail/the-top-10-causes-ofdeath.

2. National Heart, Lung, and Blood Institute. Morbidity and mortality: 2012 chart book on cardiovascular, lung and blood diseases; 2012 [cited 2018 Sep 10]. Available from: https://www. nhlbi.nih.gov/files/docs/research/2012_ChartBook_508.pdf.

3. Raherison C, Girodet PO. Epidemiology of COPD. Eur Respir Rev 2009;18(114):213-221.

4. Dalal AA, Christensen L, Liu F, Riedel AA. Direct costs of chronic obstructive pulmonary disease among managed care patients. Int J Chron Obstruct Pulmon Dis 2010;5:341-349.

5. Darnell K, Dwivedi AK, Weng Z, Panos RJ. Disproportionate utilization of healthcare resources among veterans with COPD: a retrospective analysis of factors associated with COPD healthcare cost. Cost Eff Resour Alloc 2013;11:13.

6. Crisafulli E, Costi S, Luppi F, Cirelli G, Cilione C, Coletti O, et al. Role of comorbidities in a cohort of patients with COPD undergoing pulmonary rehabilitation. Thorax 2008;63(6):487492.

7. Godtfredsen NS, Lam TH, Hansel TT, Leon ME, Gray N, Dresler $C$, et al. COPD-related morbidity and mortality after smoking cessation: status of the evidence. Eur Respir J 2008;32(4):844853.

8. Tashkin DP, Rennard S, Hays JT, Ma W, Lawrence D, Lee TC. Effects of varenicline on smoking cessation in patients with mild to moderate COPD: a randomized controlled trial. Chest 2011; 139(3):591-599.

9. Rigotti NA, Munafo MR, Stead LF. Smoking cessation interventions for hospitalized smokers: a systematic review. Arch Intern Med 2008;168(18):1950-1960.

10. Fiore MC, Jaén CR, Baker TB, Bailey WC, Benowitz NL, Curry SJ, et al. Treating tobacco use and dependence: 2008 update. Rockville: US Department of Health and Human Services. Public Health Service; 2008, 106-124.

11. Cahill K, Stevens S, Perera R, Lancaster T. Pharmacological in- terventions for smoking cessation: an overview and network meta-analysis. Cochrane Database Syst Rev 2013;(5):CD009329.

12. Anthenelli RM, Benowitz NL, West R, St Aubin L, McRae T, Lawrence $D$, et al. Neuropsychiatric safety and efficacy of varenicline, bupropion, and nicotine patch in smokers with and without psychiatric disorders (EAGLES): a double-blind, randomised, placebo-controlled clinical trial. Lancet 2016;387 (10037):2507-2520.

13. Tønnesen P. Smoking cessation and COPD. Eur Respir Rev 2013; 22(127):37-43.

14. Melzer AC, Feemster LC, Collins MP, Au DH. Predictors of pharmacotherapy for tobacco use among Veterans admitted for COPD: the role of disparities and tobacco control processes. J Gen Intern Med 2016;31(6):623-629.

15. Vaidya V, Hufstader-Gabriel M, Gangan N, Shah S, Bechtol R. Utilization of smoking-cessation pharmacotherapy among chronic obstructive pulmonary disease (COPD) and lung cancer patients. Curr Med Res Opin 2014;30(6):1043-1050.

16. Jarlenski M, Hyon Baik S, Zhang Y. Trends in use of medications for smoking cessation in medicare, 2007-2012. Am J Prev Med 2016;51(3):301-308.

17. Melzer AC, Feemster LC, Collins MP, Au DH. Utilization and effectiveness of pharmacotherapy for tobacco use following admission for exacerbation of COPD. J Hosp Med 2016;11(4): 257-263.

18. Kim MJ, Jeong IS. Smoking relapse and related factors within one year among successes of the smoking cessation clinics of public health centers. J Prev Med Public Health 2011;44(2):8492 (Korean).

19. Centers for Disease Control and Prevention. Ambulatory health care data [cited 2018 May 25]. Available from: https://www. cdc.gov/nchs/ahcd/index.htm.

20. US Food and Drug Administration (FDA). FDA Drug Safety Communication: FDA revises description of mental health side effects of the stop-smoking medicines Chantix (varenicline) and Zyban (bupropion) to reflect clinical trial findings; 2015 [cited 2018 May 25]. Available from: https://www.fda. gov/Drugs/DrugSafety/ucm532221.htm. 